\title{
Gene Expression Profile of Olfactory Transduction Signaling in an Animal Model of Human Multiple Sclerosis
}

\author{
Jeongtae Kim ${ }^{1}$, Meejung Ahn ${ }^{1}$, Yuna Choi ${ }^{1}$, Poornima Ekanayake, \\ Chul Min Park ${ }^{2}$, Changjong Moon ${ }^{3}$ Kyungsook Jung ${ }^{4}$, Akane Tanaka ${ }^{5}$, \\ Hiroshi Matsuda ${ }^{6}$ and Taekyun Shin ${ }^{1 *}$
}

${ }^{1}$ Department of Veterinary Anatomy, College of Veterinary Medicine and Veterinary Medical Research Institute, Jeju National University, Jeju 63243, ${ }^{2}$ Department of Obstetrics and Gynecology, School of Medicine, Jeju National University, Jeju 63243, ${ }^{3}$ Department of Veterinary Anatomy, College of Veterinary Medicine and BK21 Plus Project Team, Chonnam National University, Gwangju 61186, ${ }^{4}$ Immunoregulatory Materials Research Center, Korea Research Institute of Bioscience and Biotechnology, Jeongeup 56212, Korea, ${ }^{5}$ Laboratory of Comparative Animal Medicine, Division of Animal Life Science, Institute of Agriculture, Tokyo University of Agriculture and Technology, Tokyo 183-8509, ${ }^{6}$ Laboratory of Veterinary Molecular Pathology and Therapeutics, Division of Animal Life Science, Graduate School, Institute of Agriculture, Tokyo University of Agriculture and Technology, Tokyo 183-8509, Japan

\begin{abstract}
Olfactory dysfunction occurs in multiple sclerosis in humans, as well as in an animal model of experimental autoimmune encephalomyelitis (EAE). The aim of this study was to analyze differentially expressed genes (DEGs) in olfactory bulb of EAE-affected mice by next generation sequencing, with a particular focus on changes in olfaction-related signals. EAE was induced in C57BL/6 mice following immunization with myelin oligodendrocyte glycoprotein and adjuvant. Inflammatory lesions were identified in the olfactory bulbs as well as in the spinal cord of immunized mice. Analysis of DEGs in the olfactory bulb of EAE-affected mice revealed that 44 genes were upregulated (and which were primarily related to inflammatory mediators), while 519 genes were downregulated; among the latter, olfactory marker protein and stomatin-like 3 , which have been linked to olfactory signal transduction, were significantly downregulated ( $\log 2$ [fold change] $>1$ and $p$-value $<0.05$ ). These findings suggest that inflammation in the olfactory bulb of EAE-affected mice is associated with the downregulation of some olfactory signal transduction genes, particularly olfactory marker protein and stomatin-like 3, which may lead to olfactory dysfunction in an animal model of human multiple sclerosis.
\end{abstract}

Key words: Experimental autoimmune encephalomyelitis, Olfactory bulb, Olfactory marker protein, Differentially expressed gene

\section{INTRODUCTION}

Received October 9,2018, Revised December 26, 2018,

Accepted January 18,2019

* To whom correspondence should be addressed. TEL: 82-64-754-3363, FAX: 82-64-756-3354 e-mail:shint@jejunu.ac.kr
Odor perception is important for human life because every life lives with the support of odor discrimination. The olfactory bulb, which is a component of the central nervous system (CNS), processes odor signals that originate from olfactory nerves and the 
olfactory epithelium, where odor perception is transmitted by olfaction-related signals, including olfactory marker protein (OMP) $[1,2]$. In addition to OMP, a variety of olfaction-related signals are involved in odor perception in mammals, including adenyl cyclase III, stomatin, stomatin-like 3, and receptor transporter proteins [35].

Olfactory dysfunction is common with aging and is an early warning sign of neurodegenerative diseases [6], including Alzheimer's and Parkinson's disease [7] and human multiple sclerosis (MS) [8]. It has been suggested that the olfactory deficit in neurodegenerative diseases may involve structural abnormalities of the olfactory epithelium, olfactory nerves, olfactory bulb, and central olfactory cortices, as well as changes in their secondary targets [ 9 , $10]$, possibly through interruption of signal transduction molecules.

The olfactory bulb, which includes the axons of olfactory receptor neurons, is an important structure in olfactory dysfunction [11] being the major organ involved in odor processing. MS patients suffer from a loss of olfactory acuity based on olfactory functional testing using the Threshold-Discrimination-Identification (TDI) score [12-14]. Olfactory dysfunction occurs as a result of axonal degeneration in primary progressive MS patients, and CNS inflammation in relapsing-remitting MS patients [13]. In neurodegenerative diseases, microglial activation in the olfactory bulb is thought to be a consequence of CNS inflammation $[15,16]$, which also partially interrupts neural transmission [17]. Microglial activation has been also shown in the olfactory bulb of Niemann-Pick disease type $\mathrm{Cl}$ mice $[18,19]$.

Experimental autoimmune encephalomyelitis (EAE) is an animal model of human MS characterized by the infiltration of autoreactive T cells and macrophages in CNS tissues [20]. There is general agreement that, following immunization of CNS antigens in susceptible rats, auto-reactive $\mathrm{T}$ cells and bystander monocytes infiltrate the subarachnoid space, followed by meningitis and subsequent spinal cord inflammation [21]. Meningeal inflammation is immediately obvious in the caudal subarachnoid space in myelin oligodendrocyte glycoprotein (MOG)-EAE-affected mice [17] and myelin basic protein-immunized EAE-affected rats [21], and was found to extend to the cerebral cortex in the caudal-to-rostral direction, followed by parenchymal inflammation in the brain cortex [17] which may include the olfactory bulb. Considering that the brain, including the cerebrum and olfactory bulb, is chronically affected by neuroinflammation in human MS $[8,11,22]$, a chronic demyelination mouse model may be useful in the study of olfactory dysfunction.

EAE with chronic demyelination is induced in susceptible C57BL/6 mice following immunization of MOG [17]. EAE is initially characterized by infiltration of auto-reactive $\mathrm{T}$ cells in the subarachnoid space of the lower spinal cord, followed by perivascular cuffing in the spinal cord that extends its pathology in a caudo-rostral direction before reaching to the brain cortex; subventricular lesions in the cerebrum and cerebellum may also be present [17]. Axonal injury has been reported in chronic EAE lesions [23], similar to human MS brains [24]. Because some MS patients suffer from olfactory disorders $[14,25]$ that result in reduced volume of the olfactory bulb [8] and demyelination in the olfactory bulb/tract [16], it has been suggested that the olfactory bulb in EAE is structurally affected by inflammation associated with gliosis, followed by changes in olfaction-related signals in the olfactory bulb.

The olfactory deficit in EAE mice has been linked to reduced migration of progenitor cells from the subventricular zone to the olfactory bulb [26]. Olfactory dysfunction was further demonstrated neuropathologically in rat EAE, and behaviorally in mouse MOGEAE, in our previous study [27]. However, little is known regarding the changes in olfactory transduction signaling, or the neuropathological changes in the olfactory bulb, of mice with MOGEAE. Even though both the olfactory mucosa and olfactory bulb are associated with olfaction, we herein focused on the olfactory bulb, a central olfactory processing organ, which is primarily attacked by CNS autoimmune T cells in EAE. The aim of this study was to evaluate neuropathological changes and gene expression profiles in the olfactory bulb in EAE-affected animals by analyzing differentially expressed genes (DEGs).

\section{MATERIALS AND METHODS}

\section{Animals and induction of $E A E$}

We used male and female C57BL/6 mice (10 weeks old, 20 25 g; Central Lab. Animal Inc., Seoul, Korea). Experimental animals were maintained under controlled temperature $\left(24 \pm 2^{\circ} \mathrm{C}\right)$ and humidity $(55 \pm 10 \%)$ conditions, with a $12 / 12 \mathrm{~h}$ light/dark cycle, and given free access to water and standard food. The animal protocols conformed to current international laws, and to the policies of the National Institutes of Health (NIH) Guide for the Care and Use of Laboratory Animals (NIH Publication No. 85-23, 1985, revised 1996). All experimental procedures were conducted in accordance with the Guidelines for the Care and Use of Laboratory Animals of Jeju National University (Permission No. 2017-0019). Every effort was made to minimize the number of animals used and their suffering.

Mice in the EAE group were treated with $1 \mathrm{mg} / \mathrm{ml}$ of the $\mathrm{MOG}_{35-55}$ peptide (purity $>96.44 \%$; GL Biochem Ltd., Shanghai, China) emulsified in complete Freund's adjuvant (Difco Labo- 
ratories Inc., Detroit, MI, USA) and supplemented with $5 \mathrm{mg} / \mathrm{ml}$ Mycobacterium tuberculosis H37Ra (Difco Laboratories Inc.) by subcutaneous injection into the hind flank. Pertussis toxin (500 ng; List Biological Laboratories, Inc., Campbell, CA, USA) was injected intraperitoneally at days 0 and 2 post-immunization (PI) [27]. Following immunization, the experimental animals were weighed daily and their clinical signs were monitored and scored as follows: grade 0 (G.0), no signs; G.1, floppy tail; G.2, mild paraparesis; G.3, severe paraparesis; G.4, tetraparesis; G.5, moribund condition or death; R.0, recovery; and R.1, recovery with floppy tail.

\section{Tissue preparation}

Mice were fixed by transcardial perfusion with $4 \%$ paraformaldehyde in phosphate buffer (0.1 M, pH 7.4) under deep ether anesthesia at each sampling time point, including the paralytic peak (G.3, day 23 PI) and chronic paralytic (R.1, day 83 PI) stages. The skull and spinal cord were collected at each stage. For histological examination, the skull was decalcified for 10 days using decalcifying solution (formic acid-sodium citrate method) [28]. Following decalcification, the skull, including the nasal cavity, olfactory bulb, and spinal cord, was embedded in paraffin wax, and 5 - $\mu \mathrm{m}$-thick sections were prepared using a rotary microtome (RM 2135; Leica, Nussloch, Germany). Paraffin sections were deparaffinized in xylene, dehydrated in a serial ethanol solution, and then stained with hematoxylin and eosin to evaluate inflammation. For western blot analysis, the olfactory bulb and olfactory mucosa were removed and frozen at $-80^{\circ} \mathrm{C}$ until use.

\section{Library preparation and illumina sequencing}

Total RNAs of the olfactory bulb, which were collected from different individuals in control and EAE-affected mice at the paralytic peak stage ( $n=3$ per group), were extracted using TRIzol RNA Isolation Reagent (Life Technologies, Thermo Fisher Scientific, Waltham, MA, USA). The olfactory mucosa in control and EAEaffected mice ( $\mathrm{n}=3$ per group) was used to further investigate olfactory transduction signaling at the paralytic stage. The quantity and quality of total RNA were evaluated using the Agilent 2100 Bioanalyzer RNA kit (Agilent, Santa Clara, CA, USA). The isolated RNA was processed to prepare an mRNA sequencing library using the Illumina TruSeq Stranded mRNA Sample Preparation kit (Illumina, San Diego, CA, USA) according to the manufacturer's protocol. All libraries were quantified by quantitative polymerase chain reaction using the CFX96 Real Time System (Bio-Rad, Hercules, CA, USA) and sequenced using NextSeq500 sequencers (Illumina).

\section{Preprocessing and genome mapping}

Potential sequencing adapters and raw quality bases in the low reads were trimmed using Skewer [29]. After trimming the low quality bases and sequencing adapters, the cleaned high quality reads were mapped to the reference genome using STAR software [30].

\section{Quantifying gene expression and differential expressed gene analysis}

Gene annotation of the reference genome from the UCSC genome browser database (https://genome.ucsc.edu) in GTF format was used as a gene model, and the expression values were calculated as fragments per kilobase of transcript per million fragments mapped (FPKM). The DEGs between the two selected biological conditions were analyzed by Cuffdiff software in the Cufflinks package [31].

\section{Quantitative real time RT-PCR}

Total RNA of the olfactory bulb in the control, and in paralytic and recovery stages ( $\mathrm{n}=5$ per group), was isolated with TRIzol RNA Isolation Reagent (Life Technologies, Thermo Fisher Scientific) and cDNA was prepared using ReverTra Ace- $\alpha$ (TOYOBO, Osaka, Japan). The primer sequences were as follows: Omp (203 bp), forward 5'-CTCATTGTCCCTCGCCTTG-3' and reverse 5'-GCCTATCTCCTGCCTCCA-3'; and Stoml3 (193 bp), forward 5'-ATGGAGTCGTCTATTACAGAATCTACAG-3' and reverse 5'-TCGGTGGCATCATCAAGCAAG-3'. PCR reactions were performed using the CFX96 Real Time System (Bio-Rad) using SYBR Green Supermix $(2 \times)$ (TOYOBO) as follows: 40 cycles of denaturation $\left(10 \mathrm{~s}, 95^{\circ} \mathrm{C}\right)$, annealing $\left(10 \mathrm{~s}, 58^{\circ} \mathrm{C}\right)$, and extension $(10$ s, $\left.72^{\circ} \mathrm{C}\right)$.

\section{Immunohistochemistry and immunofluorescence}

Following deparaffinization, sections were prepared as previously described [32]. Briefly, following incubation with matched blocking serum $(10 \%[\mathrm{v} / \mathrm{v}]$ normal goat serum in phosphatebuffered saline [PBS]; Vectastain Elite ABC kit; Vector Laboratories, Burlingame, CA, USA), the samples were incubated with rabbit anti-ionized calcium binding protein-1 (Iba-1) (1:1,000, 019-19741, Lot. LKH4161; Wako Pure Chemical Industries, Ltd., Osaka, Japan) and mouse monoclonal anti-glial fibrillary acidic protein (GFAP) (1:400, MAB360, Lot. 2041104; EMD Millipore Corp., Temecula, CA, USA) for $1 \mathrm{~h}$ at room temperature. After three washes in PBS, the peroxidase reaction was developed using a diaminobenzidine substrate kit (Vector Laboratories).

To confirm the GFAP-positive cells in the olfactory bulb, we performed double immunofluorescence staining as in our previ- 
ous study [33]. For double staining, the sections were incubated with mouse anti-GFAP antibody, which were visualized with antimouse IgG conjugated to tetramethyl rhodamine isothiocyanate (TRITC) (1:50, T5393, lot 103K9165; Sigma-Aldrich, St, Louis, MO, USA). The sections were washed three times with PBS and then incubated with rabbit anti-glutamic acid decarboxylase (GAD) 65/67 (1:1,000, AB1511, lot 2324512; Millipore, Temecula, CA, USA) and visualized using anti-rabbit IgG conjugated fluorescein isothiocyanate (FITC) (1:50, F0382, lot 018K60572; SigmaAldrich). Images were captured using a BX-51 fluorescence microscope (Olympus, Tokyo, Japan) and merged using Adobe Photoshop (Adobe Systems, San Jose, CA, USA).

\section{Statistical analysis}

All measurements represent averages of three independent experiments. All values are presented as means \pm standard error of the mean. The results were analyzed using one-way analysis of variance followed by Student-Newman-Keuls post hoc test for multiple comparisons. A p-value $<0.05$ was considered to reflect statistical significance.

\section{RESULTS}

\section{Clinical scores and histological changes following immuni- zation}

Following immunization with the $\mathrm{MOG}_{35-55}$ peptide in mice, tail atony was observed at approximately day 12 PI. Hind limb paralysis followed at days 18 24 PI. The tail atony lasted for a considerable period. By day 30 PI, EAE-affected mice had recovered from hind limb paralysis and showed chronic paralysis (Fig. 1).

Greater inflammatory cell infiltration was detected in the spinal

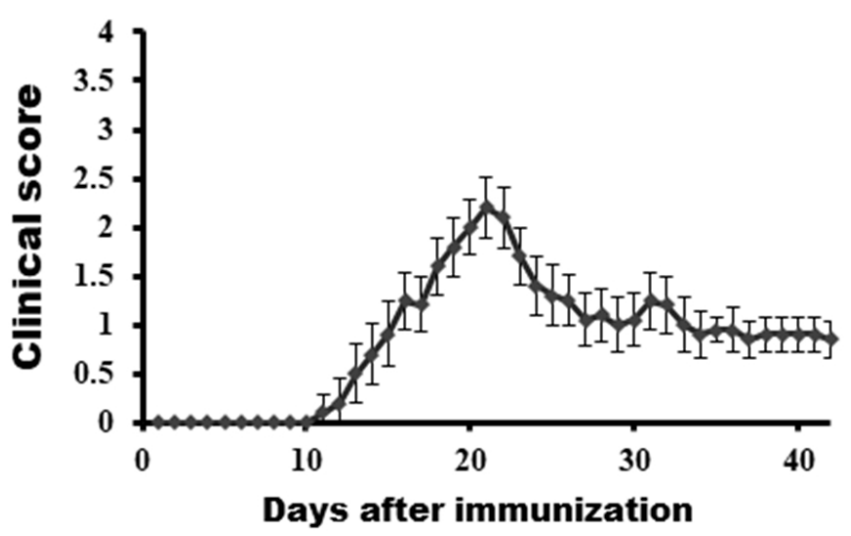

Fig. 1. Clinical scores in mice immunized with myelin oligodendrocyte glycoprotein (MOG). Immunized animals were observed daily for clinical signs of MOG-experimental autoimmune encephalomyelitis (EAE). The scores are presented as means \pm standard error of the mean (SEM). cord (Fig. 2B) and olfactory bulb (Fig. 2D) of EAE-affected mice compared with controls (Fig. 2A and 2C). Furthermore, microgliosis and astrogliosis in the olfactory bulb of EAE-induced mice were remarkably high (Fig. 2E and 2G) compared with control mice (Fig. 2F and 2H). Some Iba-1-positive cells were observed along the olfactory nerve (Fig. 2F), while the decalcified tissue did not show Iba-1-positive cells in the normal olfactory epithelium (Fig. 2E). Thus, the olfactory bulb of EAE-affected mice is significantly injured following immunization, and may be influenced by inflammatory mediators in the cerebrospinal fluid, as well as by inflammatory cells.

\section{Identification of DEGs in the olfactory bulb}

We screened for DEGs in the olfactory bulb of control and EAEaffected mice at the paralytic stage. According to the cut-off value for DEG screening ( $\log 2$ [fold change] $>1$ and $p$-value $<0.05$ ), the expression levels of 563 genes were significantly changed (44 upregulated and 519 downregulated). These altered genes may be involved in the Gene Ontology terms biological process (588), cellular component (69), or molecular function (51); CORUM protein complexes (4); human phenotype ontology (97); Kyoto Encyclopedia of Genes and Genomes (KEGG) pathways (50); miRBase microRNAs (14); or TRANSFAC TFBS (143) (Supplementary Data 1). Hierarchical clustering heat maps of the DEGs are shown in Fig. 3. We further analyzed inflammation and olfactory transduction signaling in the olfactory bulb.

\section{Profile of DEGs in inflammatory markers}

Gene expression of Il1b, Ccl2, Cd86, Stat1, and Irf5, markers of the M1 macrophage phenotype, was significantly increased (Table 1). M2 macrophage marker genes, including Trem 2 and Cd163 were also upregulated. We further examined the expression of interleukins, which have been associated with EAE pathogenesis [34]. Some interleukins (although not the majority), including Illa, Illb, and Illsbp, were significantly upregulated (data not shown).

\section{Profile of DEGs in olfactory ensheathing cell}

We further analyzed DEG for markers of olfactory ensheathing cells (OECs) $[35,36]$. The p75Ngfr was significantly downregulated, while Gfap was significantly upregulated (Table 2). With double immunofluorescence staining, most of the increased GFAP was seen in astrocytes and the olfactory nerve layer, although it was also expressed in few interneurons (Fig. 4).

\section{Profile of DEGs in olfactory transduction signaling}

Since EAE-affected mice showed olfactory dysfunction in a previous study [27], we analyzed olfactory transduction signal- 


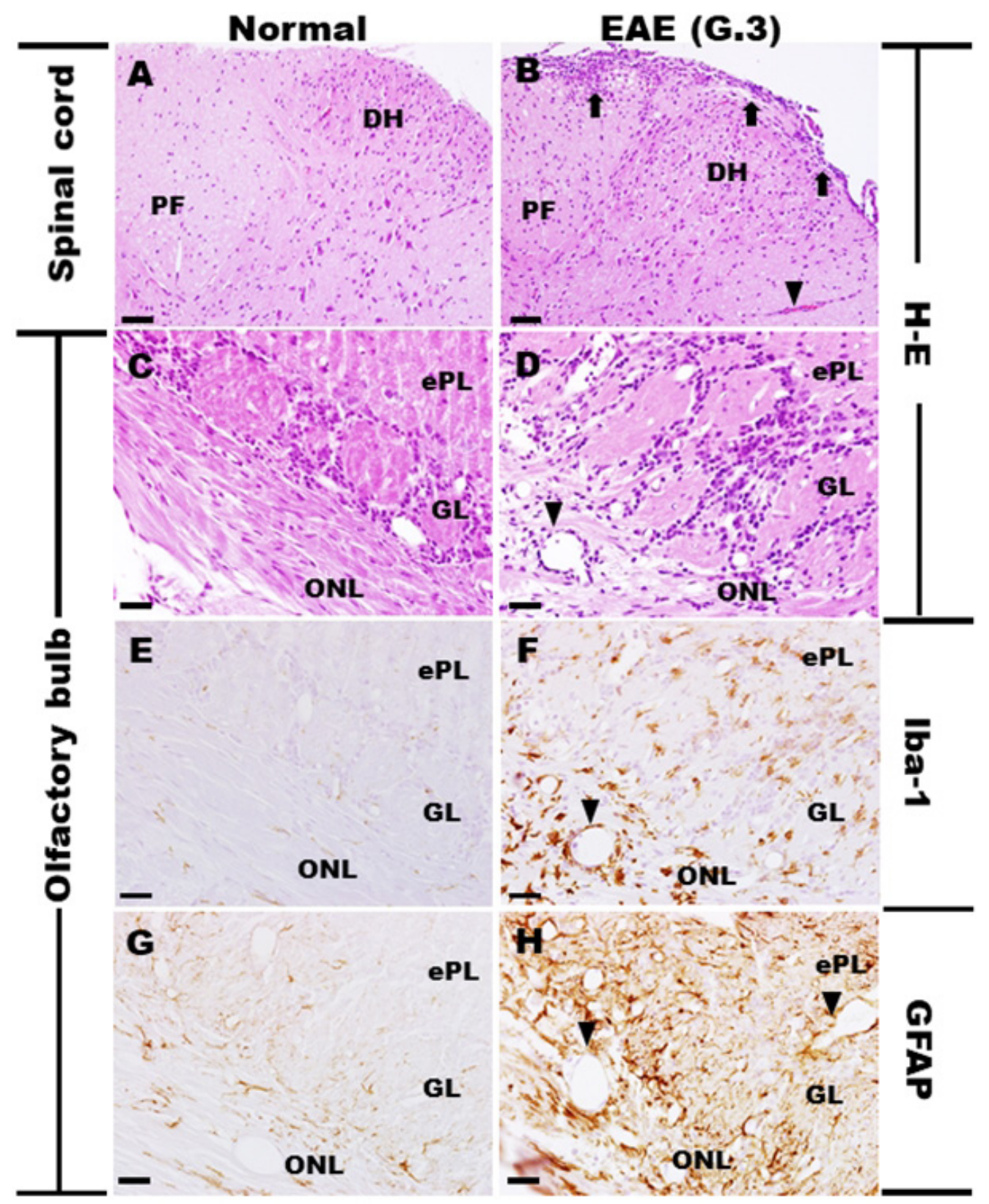

Fig. 2. Histological examination of the spinal cord and olfactory bulb of control and EAE-affected mice (G.3, paralytic stage). (A) No inflammatory cells were present in the spinal cord of control mice. (B) In EAE-affected mice, inflammatory cells (arrow) and perivascular cuffing (arrowhead) were detected in the spinal cord. (C) In control mice, there was no inflammatory response in the olfactory bulb. (D) Inflammatory cells were identified in the olfactory bulb of EAE-affected mice. Some Iba-1-positive microglia (E) and glial fibrillary acidic protein (GFAP)-positive astrocytes (G) were detected in the olfactory bulb of the control. Iba-1-positive microglia (F) and GFAP-positive astrocytes (H) were increased in the olfactory bulb of EAE-affected mice. (A D) Hematoxylin and eosin staining. (E and F) Iba-1 immunostaining. (G and H) GFAP immunostaining. Counterstained with hematoxylin. ePL, external plexiform layer; GL, glomerulus layer; GrL, granular cell layer; ML, mitral cell layer; ONL, olfactory nerve layer. Arrowheads, perivascular cuffing; arrows, inflammatory cells. Scale bars $=50 \mu \mathrm{m}$.

ing (KEGG pathway: mmu04740). Olfactory receptor genes were not detected in the olfactory bulbs (data not shown). Among 15 upregulated genes, Rtp4 was significantly increased. Among 29 downregulated genes, Omp was significantly decreased (Fig. 5A). In addition, the Stoml 3 gene, which is involved in olfactory trans- duction [3], was also significantly decreased in the olfactory bulb of EAE-affected mice. Downregulation of Omp and Stoml3 was confirmed using quantitative RT-PCR (Fig. 5B and 5C). 


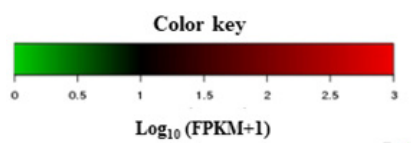

\section{Control vs EAE}

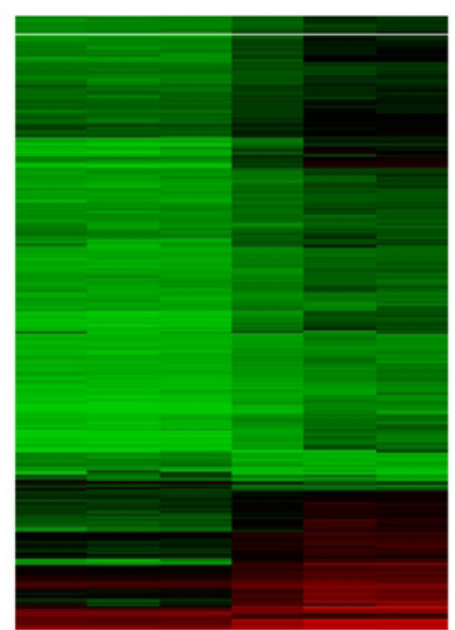

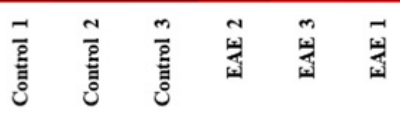

Fig. 3. Heatmap of differentially expressed genes (DEGs) in the olfactory bulbs of control versus EAE mice at the paralytic stage. Green and red indicate low and high expression, respectively.

\section{Profile of DEGs in olfactory mucosa}

We focused on the evaluation of olfactory transduction signaling-related genes (KEGG pathway: mmu04740) in the olfactory mucosa and, in particular, whether similar changes in olfactory transduction signaling-related genes occurred in the olfactory mucosa. In the olfactory mucosa of EAE-affected mice, inflammation-related genes, including $\mathrm{Il} 1 \mathrm{~b}, \mathrm{Ccl} 2$, and $\mathrm{Cd} 86$, were upregulated (data not shown), as in the olfactory bulb. In total, 120 genes, including 119 olfactory receptors and Slc8a1, were significantly increased in the olfactory mucosa of EAE-affected mice, while Gng13 and Calml3 were significantly decreased in EAE-affected mice. In addition, we also found that odorant-binding proteins, including Obpla, Obplb, and Obp2a, which are important in the odorant perception process, were significantly decreased in the olfactory mucosa of EAE-affected mice (Fig. 6A and 6B).

\section{DISCUSSION}

This is the first study to show that a number of genes involved in olfactory transduction signaling are downregulated, while inflammation-related genes are upregulated, in the olfactory bulb of EAE-affected mice.

Histopathological findings in the olfactory bulb of EAE-affected mice revealed that infiltration of inflammatory cells and subsequent microgliosis were evident; this has also been shown in the
Table 1. Pro- and anti-inflammatory differentially expressed genes in the olfactory bulb of control versus EAE paralytic stage mice

\begin{tabular}{ccccc}
\hline Description & $\begin{array}{c}\text { Participat- } \\
\text { ing genes } \\
\text { (Gensel } \\
\text { and Zhang, } \\
\text { 2015) }\end{array}$ & $\begin{array}{c}\text { log2 } \\
\text { (FC) }\end{array}$ & $\begin{array}{c}\text { Up/ } \\
\text { down }\end{array}$ & p-value \\
& Illb & 4.302 & Up & 0.023 \\
Pro-inflammatory & Cd2 & 2.301 & Up & 0.002 \\
markers (M1 phenotype) & Cd86 & 1.756 & Up & 0.00005 \\
& Stat1 & 1.631 & Up & 0.00005 \\
& Irf5 & 1.258 & Up & 0.009 \\
Anti-inflammatory & Trem2 & 1.387 & Up & 0.00005 \\
markers (M2 phenotype) & Cd163 & 1.819 & Up & 0.00005 \\
\hline
\end{tabular}

FC, fold change.

Table 2. Differentially expressed genes of olfactory ensheathing cells in the olfactory bulb of control versus EAE paralytic stage mice

\begin{tabular}{cccc}
\hline Participating genes & $\log 2(\mathrm{FC})$ & Up/down & p-value \\
\hline Gfap & 1.562 & $\mathrm{Up}^{\mathrm{a}}$ & 0.00005 \\
p75Ngfr & -1.074 & Down & 0.00005 \\
\hline
\end{tabular}

FC, fold change. ${ }^{\text {a }}$ Glial fibrillary acidic protein (GFAP) is predominantly detected in astrocytes.

olfactory bulb of human MS patients [15], suggesting that olfactory deficits in both human MS and the animal model of EAE are associated with neuroinflammation in the olfactory bulb, a central region of olfaction. Alternatively, reduced migration of progenitor cells to the olfactory bulb has been suggested as a cause of olfactory deficit in EAE-affected mice [26]. We postulate that neuroinflammation and reduced renewal of progenitor cells in the olfactory bulb is synergistically involved in the olfactory dysfunction associated with EAE. This is further supported by behavioral tests in which mice with EAE spend more time searching for buried pellets, suggesting that autoimmune CNS neuroinflammation is associated with olfactory dysfunction [27].

Next, we investigated the factors involved in the olfactory deficit in EAE through analysis of DEGs. We found that M1 phenotyperelated genes were significantly upregulated in EAE-affected mice compared with the control; M2 phenotype-related genes were upregulated in a similar manner, suggesting that both M1 and M2 phenotypes are upregulated at the paralytic stage of EAE. This upregulation was followed by downregulation of the M1 phenotype, which has also been shown in the spinal cord of EAE-affected mice [37].

Olfactory nerves are ensheathed by OECs, which ensheath olfactory nerves without myelin [35]. Embryologically, OECs arise from both the olfactory placode and neural crest $[36,38]$. As OECs have been used as a source of stem cells for transplantation, their 

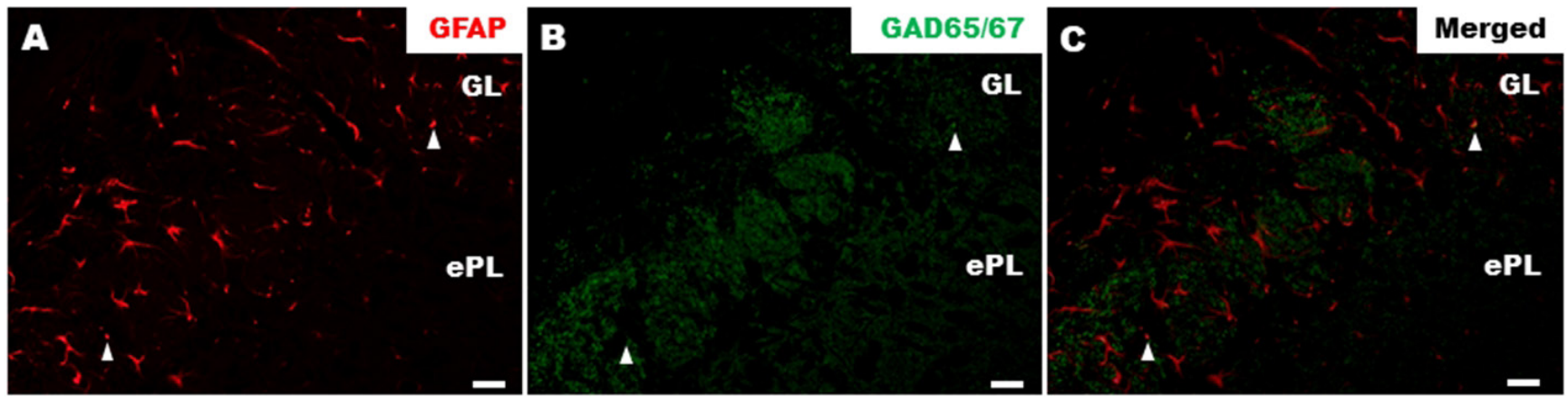

Fig. 4. Double immunofluorescence staining of GFAP (red) with interneuron markers, GAD 65/67 (green). Few GAD 65/67 positive cells (green in B) showed GFAP-positive immunoreaction (red in A) in the glomerulus layer (arrowheads in C). ePL, external plexiform layer; GL, glomerulus layer. Scale bars $=20 \mu \mathrm{m}$.
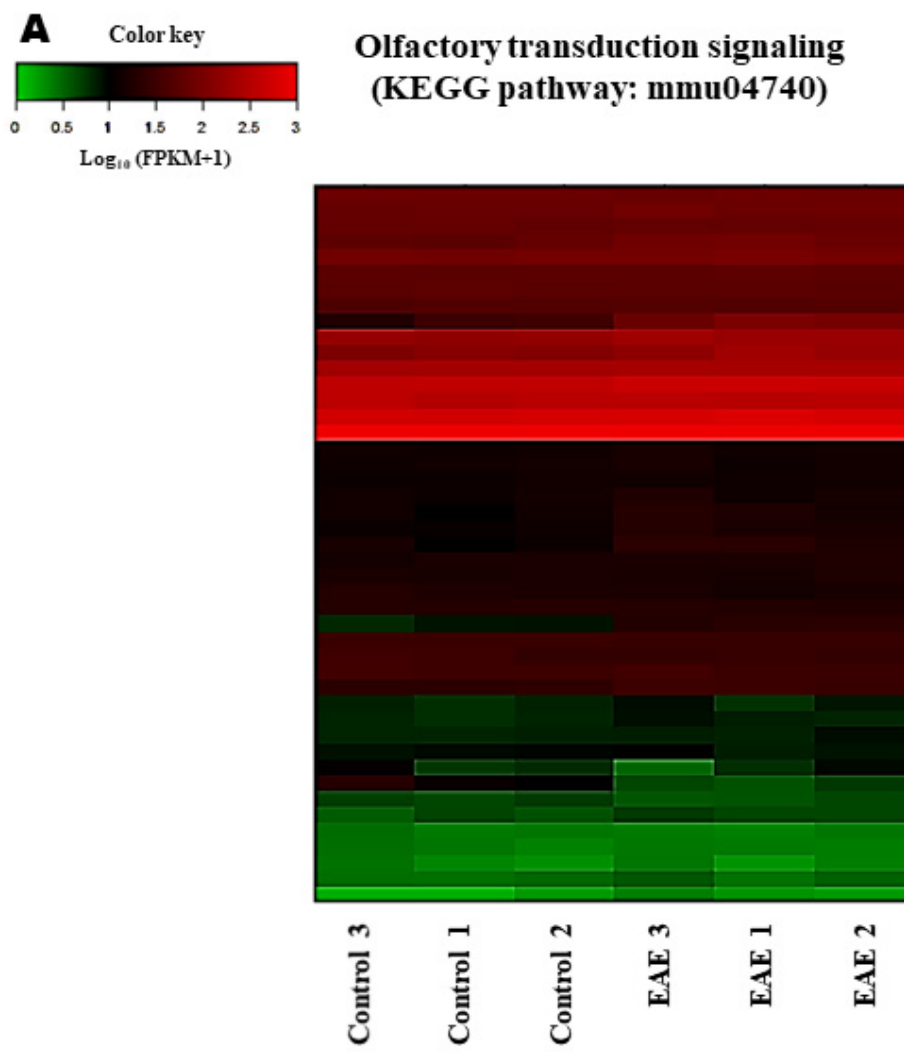

\section{Olfactory transduction signaling \\ (KEGG pathway: mmu04740)}

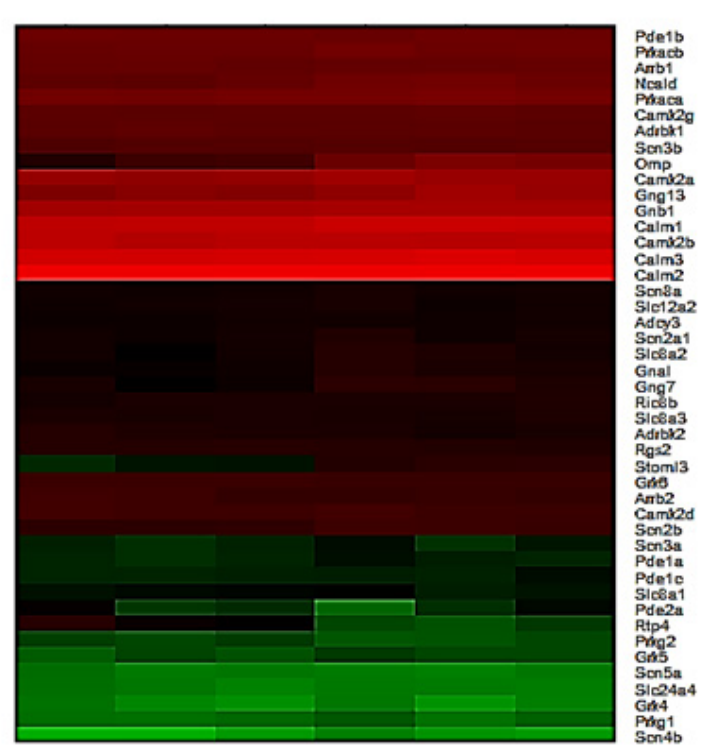

Fig. 5. Heatmap of olfactory transduction signaling (Kyoto Encyclopedia of Genes and Genomes [KEGG] pathway: mmu04740) and quantitative realtime reverse transcription-PCR (RT-PCR) of Omp and Stoml3 in the olfactory bulbs of control and EAE-affected mice. (A) Omp and Stoml3 were significantly downregulated. (B) Omp mRNA expression was significantly decreased at the paralytic stage (G.3; severe paraparesis, D23PI) and subsequently restored in the recovery stage (R.1; recovery with floppy tail, D83PI). (C) The expression level of Stoml3 mRNA was significantly decreased at the paralytic and recovery stages following immunization. PI, post-immunization. Data are presented as means \pm SEM. ${ }^{* *} \mathrm{p}<0.01$, vs. control group.

biological role requires further study [39]. In the present study, we confirmed the upregulation of GFAP, a marker of both CNS astrocytes and OECs, in the olfactory bulb of EAE-affected mice. We postulate that the increased expression of the Gfap gene is associated with astrogliosis in the olfactory bulb. However, because GFAP is occasionally expressed in Schwann cells and OECs [36],

\section{B}

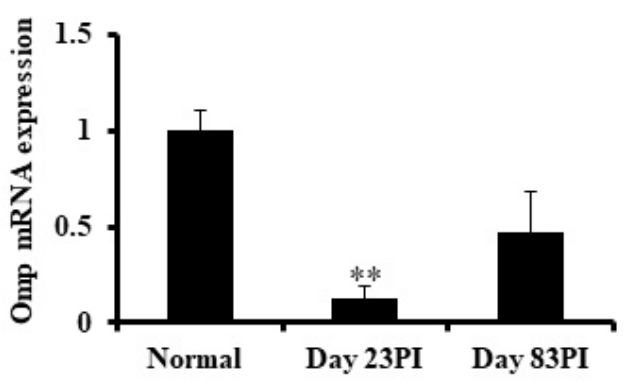

$\mathbf{C}$

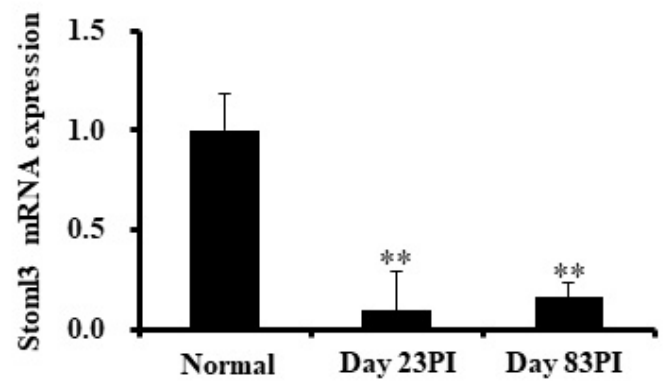



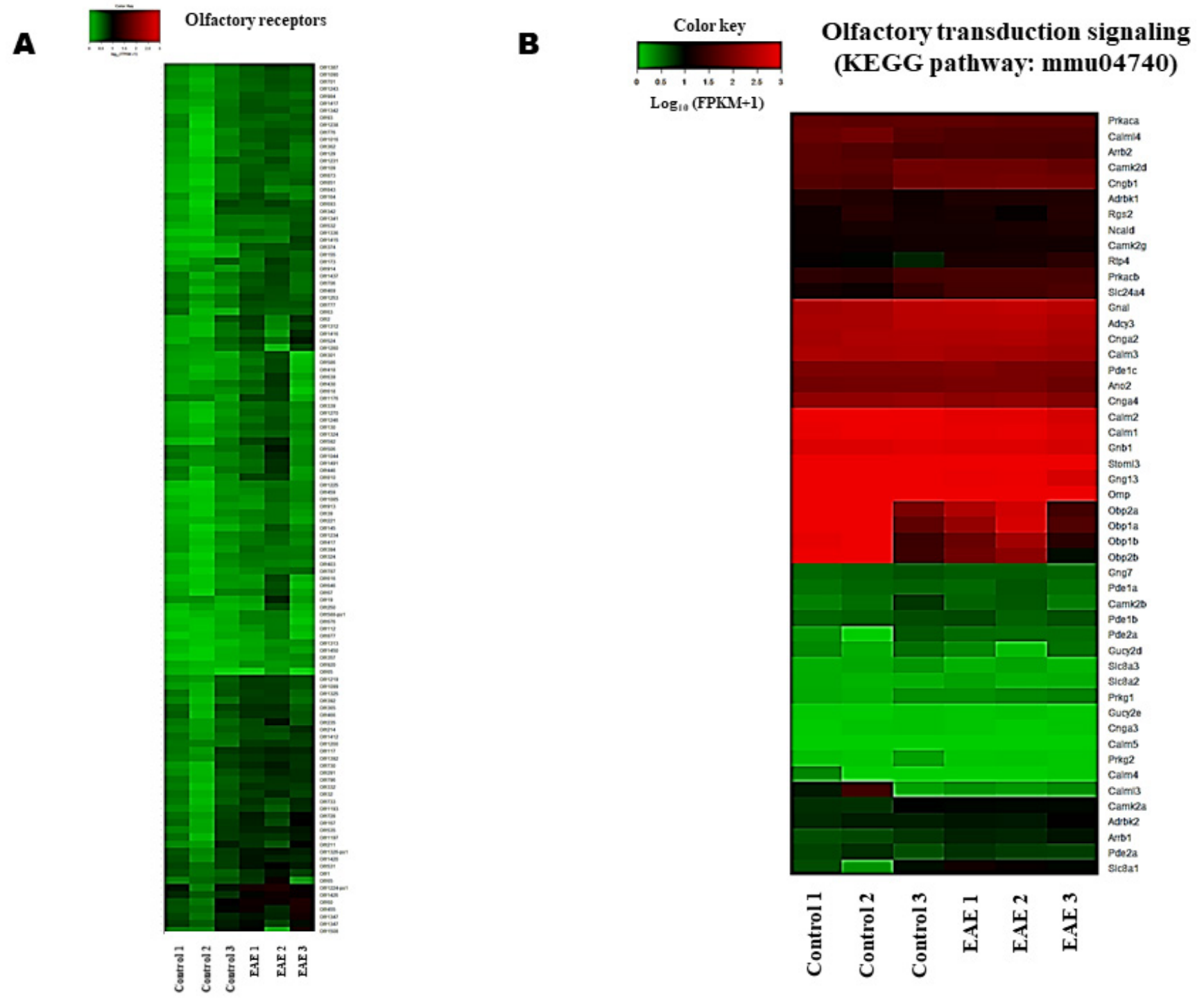

Fig. 6. Heatmap of olfactory transduction signaling (KEGG pathway: mmu04740) in the olfactory mucosa of control versus EAE mice at the paralytic stage. In total, the expression of 119 olfactory receptor genes was increased, while the expression of Gng 13 was decreased in the olfactory mucosa of EAE-affected mice.

[3]. In addition, Stoml3, alternatively named stomatin-related olfactory protein [5], was significantly downregulated, which has been associated with the dysfunction of olfaction in EAE-affected mice. Even though Rtp4 has been included in olfactory transduction signals [3], it has also been reported that Rtp4 exhibits a greater association with pro-inflammatory genes [40]. Furthermore, Rtp4 was expressed at lower levels in olfactory receptor neurons [4]. Thus, we postulate that Rtp4 is less associated with olfactory dysfunction in EAE.

The olfactory receptor in the olfactory mucosa is composed of alpha, beta, and gamma subunits of a G-coupled protein [41]. In the present study, expression level of Gng13, which is the G- protein gamma subunits, was significantly decreased. In olfactory receptor neurons, G-protein alpha stimulating olfactory type activates adenylyl cyclase type III and increases cAMP in the cilia [42]; and mice expressing mutant G-protein alpha stimulating olfactory type showed olfactory dysfunction [43]. Gng13 has been detected in the cilia of mature olfactory epithelium, and conditional knockout mice demonstrate anosmia on air-phase electro-olfactogram and the buried food test [44]. Downregulation of Gng13 was detected in both the olfactory bulb and olfactory mucosa of EAE-affected mice; thus, Gng13 appears to be strongly related to olfactory dysfunction in EAE-affected mice. Three different types of olfactory binding protein genes, including Obpla, Obp1b, and Obp2, 


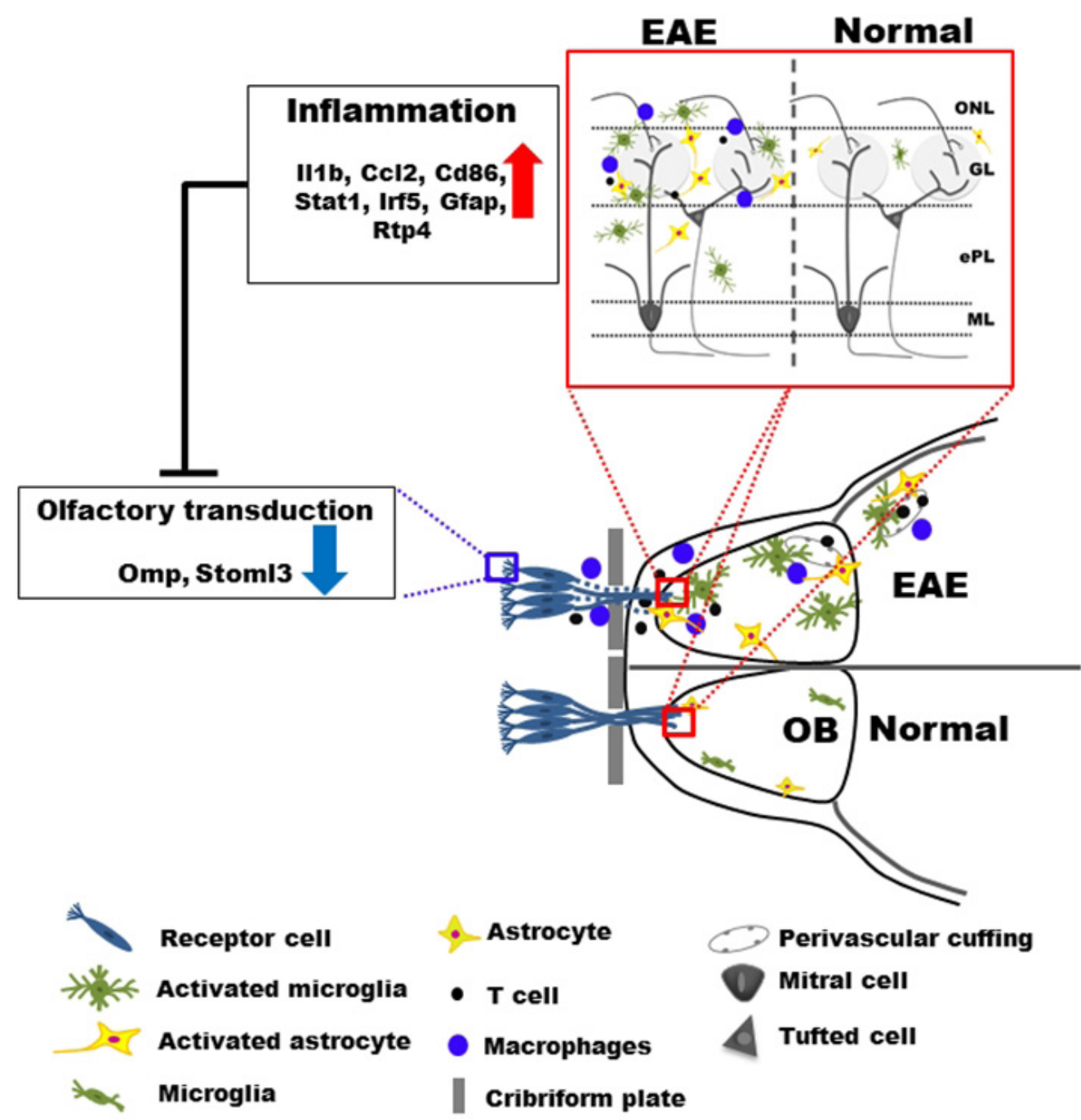

Fig. 7. Schematic illustration of the DEGs of olfactory transduction signaling and inflammatory responses in the olfactory bulb and olfactory mucosa of EAE-affected mice. At the paralytic stage, EAE-affected mice showed upregulation of inflammation-related genes and downregulation of olfactory transduction signaling in the olfactory bulb and olfactory mucosa. This schematic of neuron types in the olfactory bulb was modified from the rat nervous system [47].

were detected in the mouse [45]; these genes play an important role in the attachment of odorants to olfactory receptor cells [46]. Reduced expression of olfactory binding protein genes is thought to be a factor in the increase in olfactory receptor gene expression. These results suggest that the olfactory dysfunction seen in EAE may be closely related with disruption of the factors involved in the initiation olfactory transduction signaling, including olfactory binding protein, G-coupled protein, and olfactory receptors.

Collectively, the present findings suggest that meningeal autoimmune CNS inflammation affects the olfactory bulb and olfactory nerves, evidenced by upregulation of the inflammatory response and downregulation of myelination-related genes in the olfactory bulb of EAE-affected mice, and that neuroinflammation alters the expression of genes involved in olfactory transduction signaling, ultimately leading to olfactory dysfunction in the EAE animal model of human MS (Fig. 7).

\section{ACKNOWLEDGEMENTS}

The Authors thank Life is Art of Science Inc. (Gimpo, Republic of Korea) for next-generation sequencing. This research was supported by the National Research Foundation of Korea (Grant number: NRF-2017R1A2B4012487). 


\section{REFERENCES}

1. Dibattista M, Reisert J (2016) The odorant receptor-dependent role of olfactory marker protein in olfactory receptor neurons. J Neurosci 36:2995-3006.

2. Schild D, Restrepo D (1998) Transduction mechanisms in vertebrate olfactory receptor cells. Physiol Rev 78:429-466.

3. Kanageswaran N, Demond M, Nagel M, Schreiner BS, Baumgart S, Scholz P, Altmüller J, Becker C, Doerner JF, Conrad H, Oberland S, Wetzel CH, Neuhaus EM, Hatt H, Gisselmann G (2015) Deep sequencing of the murine olfactory receptor neuron transcriptome. PLoS One 10:e0113170.

4. Saito H, Kubota M, Roberts RW, Chi Q, Matsunami H (2004) RTP family members induce functional expression of mammalian odorant receptors. Cell 119:679-691.

5. Kobayakawa K, Hayashi R, Morita K, Miyamichi K, Oka Y, Tsuboi A, Sakano H (2002) Stomatin-related olfactory protein, SRO, specifically expressed in the murine olfactory sensory neurons. J Neurosci 22:5931-5937.

6. Hüttenbrink KB, Hummel T, Berg D, Gasser T, Hähner A (2013) Olfactory dysfunction: common in later life and early warning of neurodegenerative disease. Dtsch Arztebl Int 110:1-7, e1.

7. Doty RL (2017) Olfactory dysfunction in neurodegenerative diseases: is there a common pathological substrate? Lancet Neurol 16:478-488.

8. Goektas O, Schmidt F, Bohner G, Erb K, Ludemann L, Dahlslett B, Harms L, Fleiner F (2011) Olfactory bulb volume and olfactory function in patients with multiple sclerosis. Rhinology 49:221-226.

9. Béchade C, Cantaut-Belarif Y, Bessis A (2013) Microglial control of neuronal activity. Front Cell Neurosci 7:32.

10. Attems J, Walker L, Jellinger KA (2014) Olfactory bulb involvement in neurodegenerative diseases. Acta Neuropathol 127:459-475.

11. Caminiti F, De Salvo S, De Cola MC, Russo M, Bramanti P, Marino S, Ciurleo R (2014) Detection of olfactory dysfunction using olfactory event related potentials in young patients with multiple sclerosis. PLoS One 9:e103151.

12. Batur Caglayan HZ, Irkec C, Nazliel B, Akyol Gurses A, Capraz I (2016) Olfactory functioning in early multiple sclerosis: Sniffin’ Sticks Test study. Neuropsychiatr Dis Treat 12:21432147.

13. Schmidt FA, Maas MB, Geran R, Schmidt C, Kunte H, Ruprecht K, Paul F, Göktas Ö, Harms L (2017) Olfactory dysfunction in patients with primary progressive MS. Neurol Neuroimmunol Neuroinflamm 4:e369.
14. Lucassen EB, Turel A, Knehans A, Huang X, Eslinger P (2016) Olfactory dysfunction in multiple sclerosis: a scoping review of the literature. Mult Scler Relat Disord 6:1-9.

15. Kohl Z, Schlachetzki JC, Feldewerth J, Hornauer P, Münch M, Adame A, Riemenschneider MJ, Winkler J, Masliah E (2017) Distinct pattern of microgliosis in the olfactory bulb of neurodegenerative proteinopathies. Neural Plast 2017:3851262.

16. DeLuca GC, Joseph A, George J, Yates RL, Hamard M, Hofer M, Esiri MM (2015) Olfactory pathology in central nervous system demyelinating diseases. Brain Pathol 25:543-551.

17. Shrestha B, Jiang X, Ge S, Paul D, Chianchiano P, Pachter JS (2017) Spatiotemporal resolution of spinal meningeal and parenchymal inflammation during experimental autoimmune encephalomyelitis. Neurobiol Dis 108:159-172.

18. Seo Y, Kim HS, Kang I, Choi SW, Shin TH, Shin JH, Lee BC, Lee JY, Kim JJ, Kook MG, Kang KS (2016) Cathepsin S contributes to microglia-mediated olfactory dysfunction through the regulation of $\mathrm{Cx} 3 \mathrm{cll} 1-\mathrm{Cx} 3 \mathrm{cr} 1$ axis in a Niemann-Pick disease type C1 model. Glia 64:2291-2305.

19. Seo Y, Kim HS, Shin Y, Kang I, Choi SW, Yu KR, Seo KW, Kang KS (2014) Excessive microglial activation aggravates olfactory dysfunction by impeding the survival of newborn neurons in the olfactory bulb of Niemann-Pick disease type $\mathrm{C} 1$ mice. Biochim Biophys Acta 1842:2193-2203.

20. Shin T, Ahn M, Matsumoto Y (2012) Mechanism of experimental autoimmune encephalomyelitis in Lewis rats: recent insights from macrophages. Anat Cell Biol 45:141-148.

21. Shin T, Kojima T, Tanuma N, Ishihara Y, Matsumoto Y (1995) The subarachnoid space as a site for precursor $\mathrm{T}$ cell proliferation and effector $\mathrm{T}$ cell selection in experimental autoimmune encephalomyelitis. J Neuroimmunol 56:171-178.

22. Kapadia M, Stanojcic M, Earls AM, Pulapaka S, Lee J, Sakic B (2012) Altered olfactory function in the MRL model of CNS lupus. Behav Brain Res 234:303-311.

23. Herrero-Herranz E, Pardo LA, Gold R, Linker RA (2008) Pattern of axonal injury in murine myelin oligodendrocyte glycoprotein induced experimental autoimmune encephalomyelitis: implications for multiple sclerosis. Neurobiol Dis 30:162-173.

24. Kornek B, Storch MK, Weissert R, Wallstroem E, Stefferl A, Olsson T, Linington C, Schmidbauer M, Lassmann H (2000) Multiple sclerosis and chronic autoimmune encephalomyelitis: a comparative quantitative study of axonal injury in active, inactive, and remyelinated lesions. Am J Pathol 157:267-276.

25. Zorzon M, Ukmar M, Bragadin LM, Zanier F, Antonello RM, Cazzato G, Zivadinov R (2000) Olfactory dysfunction and extent of white matter abnormalities in multiple sclerosis: a 
clinical and MR study. Mult Scler 6:386-390.

26. Tepavčević V, Lazarini F, Alfaro-Cervello C, Kerninon C, Yoshikawa K, Garcia-Verdugo JM, Lledo PM, Nait-Oumesmar B, Baron-Van Evercooren A (2011) Inflammation-induced subventricular zone dysfunction leads to olfactory deficits in a targeted mouse model of multiple sclerosis. J Clin Invest 121:4722-4734.

27. Kim J, Choi Y, Ahn M, Jung K, Shin T (2018) Olfactory dysfunction in autoimmune central nervous system neuroinflammation. Mol Neurobiol 55:8499-8508.

28. Luna LG. Manual of histologic staining methods of the Armed Forces Institute of Pathology. 3rd ed. 1968, Blakiston Division, New York, NY.

29. Jiang H, Lei R, Ding SW, Zhu S (2014) Skewer: a fast and accurate adapter trimmer for next-generation sequencing pairedend reads. BMC Bioinformatics 15:182.

30. Dobin A, Davis CA, Schlesinger F, Drenkow J, Zaleski C, Jha S, Batut P, Chaisson M, Gingeras TR (2013) STAR: ultrafast universal RNA-seq aligner. Bioinformatics 29:15-21.

31. Trapnell C, Williams BA, Pertea G, Mortazavi A, Kwan G, van Baren MJ, Salzberg SL, Wold BJ, Pachter L (2010) Transcript assembly and quantification by RNA-Seq reveals unannotated transcripts and isoform switching during cell differentiation. Nat Biotechnol 28:511-515.

32. Ahn M, Lee C, Jung K, Kim H, Moon C, Sim KB, Shin T (2012) Immunohistochemical study of arginase- 1 in the spinal cords of rats with clip compression injury. Brain Res 1445:11-19.

33. Shin T, Kim J, Choi Y, Ahn M (2017) Glycan diversity in the vomeronasal organ of the Korean roe deer, Capreolus pygargus: a lectin histochemical study. Acta Histochem 119:778785.

34. Rangachari M, Kuchroo VK (2013) Using EAE to better understand principles of immune function and autoimmune pathology. J Autoimmun 45:31-39.

35. Borgmann-Winter K, Willard SL, Sinclair D, Mirza N, Turetsky B, Berretta S, Hahn CG (2015) Translational potential of olfactory mucosa for the study of neuropsychiatric illness. Transl Psychiatry 5:e527.

36. Ulrich R, Imbschweiler I, Kalkuhl A, Lehmbecker A, Ziege S, Kegler K, Becker K, Deschl U, Wewetzer K, Baumgärtner W (2014) Transcriptional profiling predicts overwhelming homology of Schwann cells, olfactory ensheathing cells, and Schwann cell-like glia. Glia 62:1559-1581.

37. Gensel JC, Zhang B (2015) Macrophage activation and its role in repair and pathology after spinal cord injury. Brain Res 1619:1-11.

38. Forni PE, Wray S (2012) Neural crest and olfactory system: new prospective. Mol Neurobiol 46:349-360.

39. Hoffman AM, Dow SW (2016) Concise review: stem cell trials using companion animal disease models. Stem Cells 34:1709-1729.

40. Klymenko T, Gu Q, Herbert I, Stevenson A, Iliev V, Watkins G, Pollock C, Bhatia R, Cuschieri K, Herzyk P, Gatherer D, Graham SV (2017) RNASeq analysis of differentiated keratinocytes reveals a massive response to late events during human papillomavirus type 16 infection, including loss of epithelial barrier function. J Virol (in press) 91:e01001-e01017.

41. Barbour J, Neuhaus EM, Piechura H, Stoepel N, Mashukova A, Brunert D, Sitek B, Stühler K, Meyer HE, Hatt H, Warscheid B (2008) New insight into stimulus-induced plasticity of the olfactory epithelium in Mus musculus by quantitative proteomics. J Proteome Res 7:1594-1605.

42. Choi JM, Kim SS, Choi CI, Cha HL, Oh HH, Ghil S, Lee YD, Birnbaumer L, Suh-Kim H (2016) Development of the main olfactory system and main olfactory epithelium-dependent male mating behavior are altered in Go-deficient mice. Proc Natl Acad Sci U S A 113:10974-10979.

43. Belluscio L, Gold GH, Nemes A, Axel R (1998) Mice deficient in G(olf) are anosmic. Neuron 20:69-81.

44. Li F, Ponissery-Saidu S, Yee KK, Wang H, Chen ML, Iguchi N, Zhang G, Jiang P, Reisert J, Huang L (2013) Heterotrimeric G protein subunit $G \gamma 13$ is critical to olfaction. J Neurosci 33:7975-7984.

45. Meslin C, Brimau F, Nagnan-Le Meillour P, Callebaut I, Pascal G, Monget P (2011) The evolutionary history of the SAL1 gene family in eutherian mammals. BMC Evol Biol 11:148.

46. Shiao MS, Chang AY, Liao BY, Ching YH, Lu MY, Chen SM, Li WH (2012) Transcriptomes of mouse olfactory epithelium reveal sexual differences in odorant detection. Genome Biol Evol 4:703-712.

47. Paxinos G (2015) The rat nervous system. 4th ed. Elsevier Academic Press, Amsterdam. 